\title{
New momentum and energy balance equations considering kinetic energy effect for mathematical modelling of a fixed bed adsorption column
}

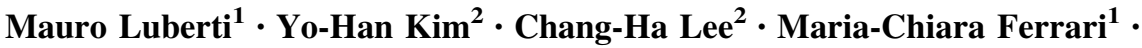 \\ Hyungwoong Ahn'
}

Received: 3 December 2014/Revised: 1 May 2015/Accepted: 30 May 2015/Published online: 11 June 2015

(C) The Author(s) 2015. This article is published with open access at Springerlink.com

\begin{abstract}
It was aimed to derive rigorous momentum and energy balance equations where the change of kinetic energy in both spatial and temporal domains of a fixed-bed adsorption column was newly taken into account. While the effect of kinetic energy on adsorption column dynamics is negligible in most cases, it can become more and more influential with an adsorption column experiencing a huge pressure drop or with the gas velocity changing abruptly with time and along the column. The rigorous momentum and energy balance equations derived in this study have been validated with two limiting cases: (1) an inert gas flow through a packed column with a very high pressure drop and (2) blowdown of an adiabatic empty column. The new energy balance including the kinetic energy effect paves a way for simulating with an improved accuracy a Rapid Pressure Swing Adsorption process that inherently involves a very high pressure drop along the column and requires very high pressure change rates for column blowdown and pressurisation.
\end{abstract}

Keywords Numerical simulation - Adsorption process · Mathematical modelling · Energy and momentum balances · Kinetic energy - Rapid pressure swing adsorption

Hyungwoong Ahn

H.Ahn@ed.ac.uk

1 Scottish Carbon Capture and Storage Centre, Institute for Materials and Processes, School of Engineering,

The University of Edinburgh, Mayfield Road,

Edinburgh EH9 3JL, UK

2 Department of Chemical and Biomolecular Engineering, Yonsei University, Seoul, Korea

\section{List of symbols}

$\mathrm{a}_{\mathrm{P}}$

column volume $\left(\mathrm{m}^{-1}\right)$

$\mathrm{c}_{\mathrm{T}} \quad$ Total concentration $\left(\mathrm{mol} / \mathrm{m}^{3}\right)$

$\hat{\mathrm{C}}_{\mathrm{pg}}, \hat{\mathrm{C}}_{\mathrm{vg}} \quad$ Specific heat of the gas mixture (J/kg K)

$\hat{\mathrm{C}}_{\mathrm{pa}} \hat{\mathrm{C}}_{\mathrm{va}} \quad$ Specific heat of the adsorbed phase $(\mathrm{J} / \mathrm{kg} \mathrm{K})$

$\hat{\mathrm{C}}_{\mathrm{pg}, \mathrm{i}} \hat{\mathrm{C}}_{\mathrm{vg}, \mathrm{i}} \quad$ Specific heat of the pure gas component $\mathrm{i}$ $(\mathrm{J} / \mathrm{kg} \mathrm{K})$

$\hat{\mathrm{C}}_{\mathrm{ps}}, \hat{\mathrm{C}}_{\mathrm{vs}} \quad$ Specific heat of the solid (J/kg K)

$\mathrm{D}_{\mathrm{B}} \quad$ Bed diameter $(\mathrm{m})$

$\mathrm{f}_{\mathrm{PD}} \quad$ Frictional energy $\left(\mathrm{N} / \mathrm{m}^{3}\right)$

g Gravity acceleration $\left(\mathrm{m} / \mathrm{s}^{2}\right)$

$\hat{\mathrm{h}}_{\mathrm{a}} \quad$ Mass enthalpy of the adsorbed phase $(\mathrm{J} / \mathrm{kg})$

$\hat{\mathrm{h}}_{\mathrm{g}}, \hat{\mathrm{h}}, \mathrm{H} \quad$ Mass enthalpy of the gas phase $(\mathrm{J} / \mathrm{kg})$

$\hat{\mathrm{h}}_{\mathrm{s}} \quad$ Mass enthalpy of the solid $(\mathrm{J} / \mathrm{kg})$

$\hat{\mathrm{h}}_{\mathrm{st}} \quad$ Mass enthalpy of the stationary phase $(\mathrm{J} / \mathrm{kg})$

ha $\mathrm{p}_{\mathrm{p}} \quad$ Heat transfer coefficient between the gas and solid phases $\left(\mathrm{W} / \mathrm{m}^{3} \mathrm{~K}\right)$

$\left(-\Delta \mathrm{H}_{\mathrm{i}}\right) \quad$ Heat of adsorption of component $\mathrm{i}(\mathrm{J} / \mathrm{mol})$

$\mathrm{h}_{\mathrm{w}} \quad$ Film heat transfer coefficient between the gas phase and the column wall $\left(\mathrm{W} / \mathrm{m}^{2} \mathrm{~K}\right)$

$\mathrm{J}_{\mathrm{T}} \quad$ Thermal flux $\left(\mathrm{W} / \mathrm{m}^{2}\right)$

$\mathrm{k}_{\mathrm{z}} \quad$ Thermal axial dispersion $(\mathrm{W} / \mathrm{m} \mathrm{K})$

L Bed length (m)

$\dot{m} \quad$ Mass flowrate $(\mathrm{kg} / \mathrm{s})$

$\bar{M} \quad$ Averaged molecular weight $(\mathrm{kg} / \mathrm{mol})$

$\mathrm{M}_{\mathrm{i}} \quad$ Molecular weight of component i $(\mathrm{kg} / \mathrm{mol})$

$\mathrm{N}_{\mathrm{c}} \quad$ Number of components

$\mathrm{P} \quad$ Total pressure (bar)

$\dot{Q} \quad$ Heat flow (W)

$\bar{q}_{i} \quad$ Pellet averaged adsorbed phase concentration $\left(\mathrm{mol} / \mathrm{m}^{3}\right)$

$\mathrm{R} \quad$ Ideal gas constant $(\mathrm{J} / \mathrm{mol} \mathrm{K})$ 
t Time (s)

T Temperature (K)

$\mathrm{T}_{\text {ref }} \quad$ Reference temperature $(\mathrm{K})$

$\mathrm{T}_{\mathrm{g}} \quad$ Gas phase temperature $(\mathrm{K})$

$\mathrm{T}_{\mathrm{s}} \quad$ Solid temperature $(\mathrm{K})$

$\mathrm{T}_{\text {st }} \quad$ Stationary phase temperature $(\mathrm{K})$

$\mathrm{T}_{\mathrm{w}} \quad$ Wall temperature $(\mathrm{K})$

$\mathrm{u} \quad$ Interstitial velocity $(\mathrm{m} / \mathrm{s})$

$\hat{U}_{a} \quad$ Mass internal energy of the adsorbed phase $(\mathrm{J} / \mathrm{kg})$

$\hat{U}_{g}, \hat{U} \quad$ Mass internal energy of the gas phase $(\mathrm{J} / \mathrm{kg})$

$\hat{U}_{s t} \quad$ Mass internal energy of the stationary phase $(\mathrm{J} / \mathrm{kg})$

$\hat{U}_{s} \quad$ Mass internal energy of the solid $(\mathrm{J} / \mathrm{kg})$

$U_{T} \quad$ Total internal energy $\left(\mathrm{J} / \mathrm{m}^{3}\right)$

$\mathrm{v}_{\mathrm{g}} \quad$ Superficial velocity $(\mathrm{m} / \mathrm{s})$

$\dot{W} \quad$ Shaft power (W)

$\mathrm{y}_{\mathrm{i}} \quad$ Molar fraction of component $\mathrm{i}(-)$

$\mathrm{w}_{\mathrm{i}} \quad$ Adsorbed mass of component $\mathrm{i}$ divided by adsorbent mass (-)

z Spatial dimension (m)

\section{Greek letters}

$\Gamma \quad$ Accumulation term, defined in Eq. $3 \mathrm{~b}\left(\mathrm{~kg} / \mathrm{m}^{3} \cdot \mathrm{s}\right)$

$\varepsilon \quad$ Bed void fraction excluding macropore $(-)$

$\varepsilon_{\mathrm{B}} \quad$ Bed void fraction including macropore $(-)$

$\rho, \rho_{g} \quad$ Gas density $\left(\mathrm{kg} / \mathrm{m}^{3}\right)$

$\rho_{a} \quad$ Adsorbed phase density $\left(\mathrm{kg} / \mathrm{m}^{3}\right)$

$\rho_{s} \quad$ Adsorbent density $\left(\mathrm{kg} / \mathrm{m}^{3}\right)$

$\rho_{s t} \quad$ Stationary phase density $\left(\mathrm{kg} / \mathrm{m}^{3}\right)$

\section{Introduction}

It is well known that fixed-bed adsorption processes, commonly used for gas separation and purification, exhibit significant temperature change during adsorption and desorption due to the heat of adsorption. The non-isothermal behaviour can make great impacts on the overall adsorption process performances, such as product purity and recovery. Hence, energy balances should be solved in combination with mass and momentum balances in simulating cyclic adsorption processes for predicting the adsorption dynamics accurately (Ruthven et al. 1994; Yang 1987; Suzuki 1990).

In early studies, equilibrium theory had usually been applied to adsorption process simulation where frozen states in adsorbed phases were assumed during the pressure-varying steps (Ruthven et al. 1994). In other words, the energy balances in equilibrium theories do not take into account the effect of the pressure change with time. Nevertheless, the simplified energy balance equation has long since been used by a number of researchers in their numerical simulation of cyclic adsorption processes incorporating both pressurisation and blowdown steps (Ruthven 1984; Kikkinides and Yang 1993; Ahn et al. 1999; Reynolds et al. 2006; Huang et al. 2008).

Meanwhile, several researchers have included mathematical terms describing the pressure change with time in the energy balance in order to simulate the pressure-varying steps more accurately (Da Silva et al. 1999, 2001; Ribeiro et al. 2008; Kim et al. 2006). Furthermore, a separate momentum equation, e.g. Ergun equation, relating to the pressure drop along the column has been incorporated in addition to the mass and energy balances. However the effects of kinetic energy on adsorption dynamics have been neglected, so the terms relating to kinetic energy change have never been included in both momentum and energy balances so far.

Since the adsorption dynamics of pressurisation and blowdown steps are more complicated than those of adsorption and purge steps due to the pressure change with time, they have been investigated by several studies in depth (Sereno and Rodrigues 1993; Rodrigues et al. 1991; Lu et al. 1992a, b). Among them, Sereno and Rodrigues (1993) investigated the effect of different forms of momentum balance, such as unsteady-state equation including kinetic energy effect, unsteady-state equation excluding kinetic energy effect, and steady-state equation (i.e. Ergun equation) during the pressurisation step. They concluded that the steady-state momentum balance (Ergun equation) could be safely used for simulating pressurisation step. However, this study was performed under isothermal condition without deriving and solving an energy balance including the kinetic energy effect. Walton and LeVan (2005) developed very general mass and energy balances applicable to multi-component fixed-bed adsorption processes, paying a good attention to thermodynamic paths of the flux terms. However, the kinetic energy terms were not incorporated into the energy balance in this study either. Therefore, deriving general momentum and energy balance equations for a fixed bed adsorption column is worth revisiting in order to identify terms that must be added to the commonly used energy balances to see the effect of kinetic energy on adsorption dynamics.

More than one energy balance equation can be used in actual numerical simulation depending on how to define the phases, such as gas, adsorbed, and solid phases. But in this study only one overall energy balance was utilised by combining the two energy balances in the gas and stationary phases under an assumption that the adsorbent, 
adsorbed gas and gas phases are at their thermal equilibrium in the control volume (Mahle et al. 1996; Richard et al. 2009).

A commercial adsorption simulator, Aspen Adsim (2004), has widely been used in both industry and academia (Kostroski and Wankat 2006; Sharma and Wankat 2009; Farzaneh et al. 2013). The energy balance formulae used in Adsim were written in its reference guide. As the energy balance equations in Adsim appear to have been derived taking into account the change of the kinetic energy, their relevance was also examined in the Appendix.

\section{Derivation of the momentum and energy balance equations with kinetic energy effects}

In this study the derivation of the momentum and energy balance equations for a fixed bed adsorption column was carried out under the following assumptions:

(1) Internal energy accumulates in the gas and stationary phases.

(2) Enthalpy is convected in the gas phase only.

(3) Kinetic energy accumulates and is convected in the gas phase only.

(4) Thermal energy dispersion in the gas phase is included. But axial thermal dispersion in the stationary phase is neglected assuming that there would not be such a great temperature gradient inside the particle.

(5) Enthalpy and kinetic energy disappear (or appear) by adsorption (or desorption) in the gas phase. On the contrary, only enthalpy appears (or disappears) in the stationary phase by adsorption (or desorption). The adsorption (or desorption) involves the heat of adsorption in the stationary phase in addition to the enthalpy change by mass addition (or reduction).

(6) Heat transfer through the column wall in the gas phase is included.

(7) Heat is transferred between the two phases.

(8) Ideal gas law applies to the gas phase.

(9) All the properties are homogeneous over a phase: there is no difference in a property between the bulk and the boundary within a phase.

(10) The drag force in the gas phase is included. The gas phase drag force is transferred to the solid phase and subsequently dissipated to the wall in the form of static friction.

(11) There is no accumulation of extensive properties in the boundary.

(12) Adsorbed phase is not deemed as mobile phase but stationary phase.
(13) Potential energy is negligible.

(14) Bed void fraction is kept constant with respect to both time and space.

(15) Turbulent heat flux is neglected.

(16) There is no interfacial shear stress.

Most of all, the mass balances in the gas and stationary phases are established as follows since they will be often used in the derivation of the momentum and energy balances.

Mass balance in the gas phase (molar):

$\varepsilon \frac{\partial c_{T}}{\partial t}+\varepsilon \frac{\partial\left(c_{T} u\right)}{\partial z}+(1-\varepsilon) \cdot \sum_{i=1}^{N_{c}} \frac{\partial \bar{q}_{i}}{\partial t}=0$

In mass terms:

$\varepsilon \frac{\partial \rho_{g}}{\partial t}+\varepsilon \frac{\partial\left(\rho_{g} u\right)}{\partial z}=\Gamma$

where

$\rho_{g}=c_{T} \bar{M}$

$\Gamma=-(1-\varepsilon) \cdot \sum_{i=1}^{N_{c}} \frac{\partial\left(\bar{q}_{i} M_{i}\right)}{\partial t}$

The average molecular weight, $\bar{M}$, in Eq. (3a) is defined by

$\bar{M}=\sum_{i=1}^{N_{c}} y_{i} M_{i}$

Accordingly the mass balance in the stationary phase in mass terms is

$(1-\varepsilon) \frac{\partial \rho_{a}}{\partial t}=-\Gamma$

Now we construct momentum and energy balance equations including the kinetic energy effect in temporal and spatial domains. All the terms being considered for constructing the energy balance in this study are shown graphically in Fig. 1.

Assumption A.1 gives the following terms:

In the gas phase,

$\varepsilon \frac{\partial\left(\rho_{g} \hat{U}_{g}\right)}{\partial t}$

In the stationary phase,

$(1-\varepsilon) \frac{d\left(\rho_{s t} \hat{U}_{s t}\right)}{d t}=(1-\varepsilon)\left[\frac{d\left(\rho_{s} \hat{U}_{s}\right)}{d t}+\frac{d\left(\rho_{a} \hat{U}_{a}\right)}{d t}\right]$

where we use the standard thermodynamic definition of the internal energy per unit volume. 


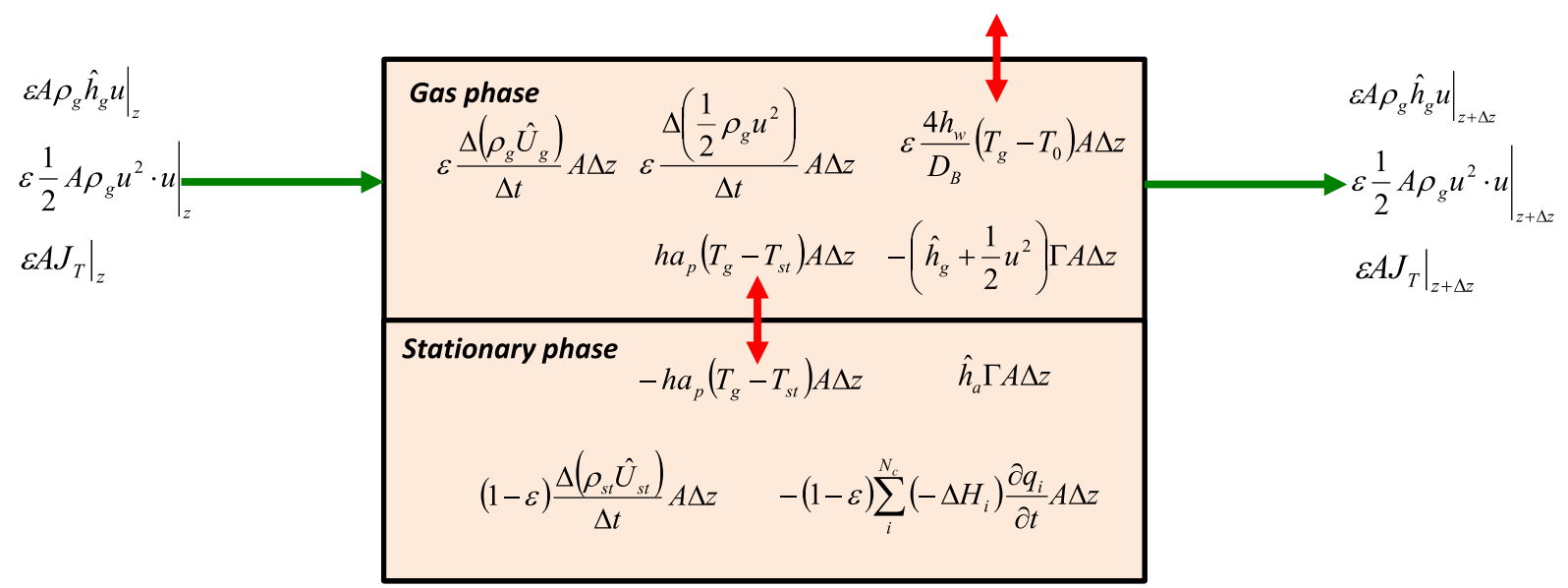

Fig. 1 Shell balance for the derivation of a rigorous energy balance equation including the kinetic energy effect in a column element. Equations (18) and (19) are made up of the terms in the figure

$\hat{U}_{g}=\hat{U}_{g, r e f}+\sum_{i}^{N_{c}} \int_{T_{r e f}}^{T} y_{i} \hat{C}_{v g, i} d T=\hat{U}_{g, r e f}+\int_{T_{r e f}}^{T} \hat{C}_{v g, a v e} d T$

$\hat{U}_{s} \cong \hat{h}_{s}=\hat{h}_{s, r e f}+\int_{T_{r e f}}^{T} \hat{C}_{p s} d T$

$$
\begin{aligned}
\hat{U}_{a} \cong \hat{h}_{a} & =\hat{h}_{a, r e f}+\sum_{i}^{N_{c}} \int_{T_{r e f}}^{T} y_{i} \hat{C}_{p a, i} d T \\
& =\hat{h}_{a, r e f}+\int_{T_{\text {ref }}}^{T} \hat{C}_{p a, a v e} d T
\end{aligned}
$$

A.2 gives the following term

$$
\varepsilon \frac{\partial\left(\rho_{g} \hat{h}_{g} u\right)}{\partial z}
$$

A.3 gives the following terms

$\varepsilon \frac{\partial\left(\frac{1}{2} \rho_{g} u^{2}\right)}{\partial t}+\varepsilon \frac{\partial\left(\frac{1}{2} \rho_{g} u^{2} \cdot u\right)}{\partial z}$

For simplicity we write A.4 in terms of the thermal flux, i.e. Fourier's law.

$\varepsilon \frac{\partial J_{T}}{\partial z}$

A.5 is the change of the enthalpy and the kinetic energy carried by the mass transferring between the two phases by adsorption and desorption. In addition, the heat of adsorption is generated in the stationary phase by adsorption. Assuming that phases are homogeneous (A.9), the change of the enthalpy and the kinetic energy by adsorption in each phase can be written as $-\left(\hat{h}_{g}+\frac{1}{2} u^{2}\right) \Gamma$ in the gas phase

$\hat{h}_{a} \Gamma-(1-\varepsilon) \sum_{i}^{N_{c}}\left(-\Delta H_{i}\right) \frac{\partial \bar{q}_{i}}{\partial t}$ in the adsorbed phase

A.6 can be expressed by the following standard equation.

$\frac{4 h_{w}}{D_{B}}\left(T_{g}-T_{w}\right)$

A.7 describes heat transfer between the two phases

$h a_{p}\left(T_{g}-T_{s t}\right)$

In the end the energy balance in the gas phase can be expressed by summing Eqs. (6), (11)-(14), (16), and (17):

$$
\begin{aligned}
& \varepsilon \frac{\partial\left(\rho_{g} \hat{U}_{g}\right)}{\partial t}+\varepsilon \frac{\partial\left(\rho_{g} \hat{h}_{g} u\right)}{\partial z}+\varepsilon \frac{\partial\left(\frac{1}{2} \rho_{g} u^{2}\right)}{\partial t}+\varepsilon \frac{\partial\left(\frac{1}{2} \rho_{g} u^{2} \cdot u\right)}{\partial z} \\
& -\left(\hat{h}_{g}+\frac{1}{2} u^{2}\right) \Gamma+h a_{p}\left(T_{g}-T_{s t}\right)+\varepsilon \frac{\partial J_{T}}{\partial z}+\varepsilon \frac{4 h_{w}}{D_{B}}\left(T_{g}-T_{w}\right)=0
\end{aligned}
$$

Similarly, the energy balance in the stationary phase can be written by summing Eqs. (7), (15) and (17) as:

$$
\begin{aligned}
& (1-\varepsilon) \frac{d\left(\rho_{s t} \hat{U}_{s t}\right)}{d t}+\hat{h}_{a} \Gamma-(1-\varepsilon) \sum_{i}^{N_{c}}\left(-\Delta H_{i}\right) \frac{d \bar{q}_{i}}{d t} \\
& -h a_{p}\left(T_{g}-T_{s t}\right)=0
\end{aligned}
$$

To write the momentum balance taking into account the mass transfer between the phases is not a trivial matter. We obtain the momentum balance in the gas phase by simplifying the formulation of Ishii and Hibiki (2010) taking into 
account that the void fraction is constant and neglecting internal shear stresses, i.e. inviscid fluid:

$\varepsilon \frac{\partial\left(\rho_{g} u\right)}{\partial t}+\varepsilon \frac{\partial\left(\rho_{g} u \cdot u\right)}{\partial z}+\varepsilon \frac{\partial P}{\partial z}-\Gamma u+\varepsilon f_{P D}=0$

Where the penultimate term arises due to phase change; the last term is the drag between the two phases, i.e. Ergun equation. It should be noted that the shear stress on the wall and the potential energy were neglected.

Now we convert Eq. (18) to the equivalent enthalpy balance equation. Consider first the two terms relating to the kinetic energy change in the temporal and spatial domains in Eq. (18):

$\varepsilon \frac{\partial\left(\frac{1}{2} \rho_{g} u^{2}\right)}{\partial t}+\varepsilon \frac{\partial\left(\frac{1}{2} \rho_{g} u^{2} \cdot u\right)}{\partial z}$

By expanding Eq. (21) we can write

$\varepsilon\left(\rho_{g} u \frac{\partial u}{\partial t}+\frac{u^{2}}{2} \frac{\partial \rho_{g}}{\partial t}\right)+\varepsilon\left(\rho_{g} u^{2} \frac{\partial u}{\partial z}+\frac{u^{2}}{2} \frac{\partial \rho_{g} u}{\partial z}\right)$

which can be rearranged into

$\varepsilon \rho_{g} u\left(\frac{\partial u}{\partial t}+u \frac{\partial u}{\partial z}\right)+\varepsilon \frac{u^{2}}{2}\left(\frac{\partial \rho_{g}}{\partial t}+\frac{\partial \rho_{g} u}{\partial z}\right)$

If the mass balance does not involve adsorption, the second term in Eq. (23) cancels out but in case of adsorption taking place we have an extra term, i.e.

$\varepsilon \rho_{g} u\left(\frac{\partial u}{\partial t}+u \frac{\partial u}{\partial z}\right)+\frac{1}{2} u^{2} \Gamma$

To expand the two terms inside the parenthesis relating to velocity in Eq. (24), the momentum balance in the gas phase, Eq. (20), will be used later as follows:

$$
\begin{aligned}
& \varepsilon u\left[\frac{\partial \rho_{g}}{\partial t}+\frac{\partial\left(\rho_{g} u\right)}{\partial z}\right]+\varepsilon \rho_{g}\left(\frac{\partial u}{\partial t}+u \frac{\partial u}{\partial z}\right)+\varepsilon \frac{\partial P}{\partial z}-\Gamma u \\
& +\varepsilon f_{P D}=0 \\
& \varepsilon \rho_{g} u\left(\frac{\partial u}{\partial t}+u \frac{\partial u}{\partial z}\right)=-\varepsilon u\left[f_{P D}+\frac{\partial P}{\partial z}\right]
\end{aligned}
$$

Using A.8 we can rewrite the first two terms in Eq. (18) as:

$$
\begin{aligned}
& \varepsilon\left[\frac{\partial\left(\rho_{g} \hat{U}_{g}\right)}{\partial t}+\frac{\partial\left(\left(\rho_{g} \hat{h}_{g}\right) \cdot u\right)}{\partial z}\right] \\
& =\varepsilon\left[\frac{\partial\left(\rho_{g} \hat{h}_{g}\right)}{\partial t}+\frac{\partial\left(\left(\rho_{g} \hat{h}_{g}\right) \cdot u\right)}{\partial z}-R \frac{\partial\left(\rho_{g} T / \bar{M}\right)}{\partial t}\right] \\
& =\varepsilon\left[\frac{\partial\left(\rho_{g} \hat{h}_{g}\right)}{\partial t}+\frac{\partial\left(\left(\rho_{g} \hat{h}_{g}\right) \cdot u\right)}{\partial z}\right]-\varepsilon \frac{\partial P}{\partial t}
\end{aligned}
$$

Substituting Eqs. (24), (26) and (27) into Eq. (18), we obtain the enthalpy balance equation in the gas phase:

$$
\begin{aligned}
& \varepsilon\left[\frac{\partial\left(\rho_{g} \hat{h}_{g}\right)}{\partial t}+\frac{\partial\left(\left(\rho_{g} \hat{h}_{g}\right) \cdot u\right)}{\partial z}\right]-\varepsilon \frac{\partial P}{\partial t}-\varepsilon u\left[f_{P D}+\frac{\partial P}{\partial z}\right] \\
& -\hat{h}_{g} \Gamma+h a_{p}\left(T_{g}-T_{s t}\right)+\varepsilon \frac{\partial J_{T}}{\partial z}+\varepsilon \frac{4 h_{w}}{D_{B}}\left(T_{g}-T_{w}\right)=0
\end{aligned}
$$

Note that the Eq. (28) is similar to the Ishii and Hibiki's equation on one-dimensional two phase flow (Ishii and Hibiki, 2010).

Since $\hat{U}_{s t} \cong \hat{h}_{s t}$, the energy balance equation in the stationary phase, Eq. (19), can also be deemed as an enthalpy balance equation, Eq. (29).

$$
\begin{aligned}
& (1-\varepsilon) \frac{d\left(\rho_{s t} \hat{h}_{s t}\right)}{d t}+\hat{h}_{a} \Gamma-(1-\varepsilon) \sum_{i}^{N_{c}}\left(-\Delta H_{i}\right) \frac{d \bar{q}_{i}}{d t} \\
& -h a_{p}\left(T_{g}-T_{s t}\right)=0
\end{aligned}
$$

Now we expand the two enthalpy balance equations in the gas and stationary phases further so that they can be expressed in terms of temperature instead of enthalpy.

The first two terms in LHS of Eq. (28) can be expanded assuming that the heat capacity in the gas phase is constant.

$$
\begin{array}{r}
\varepsilon \frac{\partial\left(\rho_{g} \hat{h}_{g}\right)}{\partial t}+\varepsilon \frac{\partial\left(\rho_{g} u \hat{h}_{g}\right)}{\partial z}=\varepsilon \rho_{g}\left(\frac{\partial \hat{h}_{g}}{\partial t}+u \frac{\partial \hat{h}_{g}}{\partial z}\right)+\hat{h}_{g} \Gamma \\
=\varepsilon \rho_{g} \hat{C}_{p g, a v e}\left(\frac{\partial T_{g}}{\partial t}+u \frac{\partial T_{g}}{\partial z}\right)+\hat{h}_{g} \Gamma
\end{array}
$$

Substituting Eq. (30) into the enthalpy balance equation in the gas phase, Eq. (28), the energy balance equation in the gas phase in terms of temperature is obtained as follows.

$$
\begin{array}{r}
\varepsilon \rho_{g} \hat{C}_{p g, a v e}\left(\frac{\partial T_{g}}{\partial t}+u \frac{\partial T_{g}}{\partial z}\right)-\varepsilon \frac{\partial P}{\partial t}-\varepsilon u\left[f_{P D}+\frac{\partial P}{\partial z}\right] \\
+h a_{p}\left(T_{g}-T_{s t}\right)+\frac{\partial J_{T}}{\partial z}+\varepsilon \frac{4 h_{w}}{D_{B}}\left(T_{g}-T_{w}\right)=0
\end{array}
$$

Similarly the enthalpy balance equation in the stationary phase, Eq. (29), can be converted into its equivalent energy balance equation in the stationary phase in terms of temperature, Eq. (32):

$$
\begin{aligned}
& (1-\varepsilon)\left[\rho_{s} \hat{C}_{p s} \frac{d T_{s t}}{d t}+\rho_{a} \hat{C}_{p a, a v e} \frac{d T_{s t}}{d t}-\sum_{i}^{N_{c}}\left(-\Delta H_{i}\right) \frac{d \bar{q}_{i}}{d t}\right] \\
& -h a_{p}\left(T_{g}-T_{s t}\right)=0
\end{aligned}
$$


Using

$$
\begin{gathered}
(1-\varepsilon) \frac{d\left(\rho_{s t} \hat{h}_{s t}\right)}{d t}=(1-\varepsilon)\left[\frac{d\left(\rho_{s} \hat{h}_{s}\right)}{d t}+\frac{d\left(\rho_{a} \hat{h}_{a}\right)}{d t}\right] \\
=(1-\varepsilon)\left[\rho_{s} \frac{d \hat{h}_{s}}{d t}+\rho_{a} \frac{d \hat{h}_{a}}{d t}+\hat{h}_{a} \frac{d \rho_{a}}{d t}\right] \\
=(1-\varepsilon)\left[\rho_{s} \hat{C}_{p s} \frac{d T_{s t}}{d t}+\rho_{a} \hat{C}_{p a, a v e} \frac{d T_{s t}}{d t}\right]-\hat{h}_{a} \Gamma
\end{gathered}
$$

If an instantaneous thermal equilibrium between the gas and stationary phases is assumed, an overall energy balance can be obtained by simply summing Eqs. (31) and (32).

$$
\begin{aligned}
& \varepsilon \rho_{g} \hat{C}_{p g, a v e}\left(\frac{\partial T}{\partial t}+u \frac{\partial T}{\partial z}\right)+ \\
& (1-\varepsilon)\left[\rho_{s} \hat{C}_{p s} \frac{\partial T}{\partial t}+\rho_{a} \hat{C}_{p a, a v e} \frac{\partial T}{\partial t}-\sum_{i}^{N_{c}}\left(-\Delta H_{i}\right) \frac{\partial \bar{q}_{i}}{\partial t}\right] \\
& \quad=\varepsilon k_{z, g} \frac{\partial^{2} T}{\partial z^{2}}+\varepsilon \frac{\partial P}{\partial t}+\varepsilon u \frac{\partial P}{\partial z}+\varepsilon u f_{P D}-\varepsilon \frac{4 h_{w}}{D_{B}}\left(T-T_{w}\right)
\end{aligned}
$$

Hereinafter, Eq. (34) is referred to as Rigorous model.

In case of using a simple momentum balance equation where the pressure drop along the column is determined solely by Ergun equation, the $\varepsilon u \frac{\partial P}{\partial z}+\varepsilon u f_{P D}$ in the RHS of Eq. (34) would disappear. In this study, however, the two terms cannot be cancelled out since the full momentum equation, Eq. (26), must be used in combination with the Rigorous model for coherence.

\section{Commonly used energy balances}

By and large there are two different cohorts of energy balance equations usually used in adsorption research community which have several terms missing in their formulas in comparison to Eq. (34). The first simplified energy balance, hereinafter referred to as Simplified 1 model, is generally expressed in the following equation.

$$
\begin{array}{r}
\left(\hat{C}_{p g} \rho_{g}+\frac{1-\varepsilon}{\varepsilon} \cdot \hat{C}_{p s} \rho_{s}\right) \frac{\partial T}{\partial t}-k_{z, g} \frac{\partial^{2} T}{\partial z^{2}}+\hat{C}_{p g} \rho_{g} u \cdot \frac{\partial T}{\partial z} \\
-\frac{1-\varepsilon}{\varepsilon} \cdot \sum_{i=1}^{N_{c}}\left(-\Delta H_{i}\right) \frac{\partial \bar{q}_{i}}{\partial t}+\frac{4 h_{w}}{D_{B}} \cdot\left(T-T_{w}\right)=0
\end{array}
$$

This well-known formula can be expressed in more complicated forms than Eq. (35) by taking into account the gas phase in the macropore separately, introducing the heat capacity in the adsorbed phase, and so on (Ruthven 1984; Kikkinides and Yang 1993; Reynolds et al. 2006; Huang et al. 2008). When compared to Eq. (34), the Simplified 1 model does not contain three terms, $\varepsilon \frac{\partial P}{\partial t}, \varepsilon u \frac{\partial P}{\partial z}$, and $\varepsilon u f_{P D}$. As the last two terms are relating to the kinetic energy change as shown in Eq. (26), it is obvious that the Simplified 1 model does not include the effect of kinetic energy change. Another missing term, $\varepsilon \frac{\partial P}{\partial t}$, can be omitted only in constant pressure steps, such as adsorption and purge steps.

The second simplified energy balance, hereinafter referred to as Simplified 2 model, has the following formula (Da Silva et al. 1999, 2001; Ribeiro et al. 2008; Kim et al. 2006).

$$
\begin{aligned}
& \left(\hat{C}_{v g} \rho_{g}+\frac{1-\varepsilon}{\varepsilon} \cdot \hat{C}_{p s} \rho_{s}\right) \frac{\partial T}{\partial t}-\frac{R T}{\bar{M}} \frac{\partial \rho_{g}}{\partial t}-k_{z, g} \frac{\partial^{2} T}{\partial z^{2}}+\hat{C}_{p g} \rho_{g} u \cdot \frac{\partial T}{\partial z} \\
& -\frac{1-\varepsilon}{\varepsilon} \cdot \sum_{i=1}^{N c}\left(-\Delta H_{i}\right) \frac{\partial \bar{q}_{i}}{\partial t}+\frac{4 h_{w}}{D_{B}} \cdot\left(T-T_{w}\right)=0
\end{aligned}
$$

The Simplified 2 model, Eq. (36), is similar to the Simplified 1 model, Eq. (35), but it contains $\hat{C}_{v g} \rho_{g} \frac{\partial T}{\partial t}$ instead of $\hat{C}_{p g} \rho_{g} \frac{\partial T}{\partial t}$ and also includes one additional term of $-\frac{R T}{\bar{M}} \frac{\partial \rho_{g}}{\partial t}$.

To figure out which terms are missing in comparison to the Rigorous model, we convert the Rigorous model, Eq. (34), into the following equation by simple mathematical manipulation.

$$
\begin{aligned}
& \varepsilon \rho_{g} \hat{C}_{v g, a v e} \frac{\partial T}{\partial t}+\varepsilon \rho_{g} \hat{C}_{p g, a v e} u \frac{\partial T}{\partial z} \\
& +(1-\varepsilon)\left[\rho_{s} \hat{C}_{p s} \frac{\partial T}{\partial t}+\rho_{a} \hat{C}_{p g, a v e} \frac{\partial T}{\partial t}-\sum_{i}^{N_{c}}\left(-\Delta H_{i}\right) \frac{\partial q_{i}}{\partial t}\right] \\
& =\varepsilon k_{z, g} \frac{\partial^{2} T}{\partial z^{2}}+\varepsilon \frac{R T}{\bar{M}} \frac{\partial \rho_{g}}{\partial t}+\varepsilon u \frac{\partial P}{\partial z}+\varepsilon u f_{P D}(\varepsilon, u)-\varepsilon \frac{4 h_{w}}{D_{B}}\left(T-T_{w}\right)
\end{aligned}
$$

Two terms, such as $\varepsilon u \frac{\partial P}{\partial z}$ and $\varepsilon u f_{P D}$, do not appear in the Simplified 2 model in comparison to another form of the Rigorous model, Eq. (37). As they are relating to the kinetic energy change, it can be concluded that the Simplified 2 model does not consider the kinetic energy change in common with the Simplified 1 model.

In addition, Aspen Adsim appears to use an energy balance equation different from the abovementioned two simplified equations according to its reference guide (Aspen Adsim 2004). A detailed review on the validity of the energy balance equation that Aspen Adsim uses was given in the Appendix separately. 


\section{Validation of the rigorous energy balance with two limiting cases}

The Rigorous model is distinguished from the two simplified models in terms of (1) including the effect of kinetic energy change in the temporal and spatial domains and (2) in common with the Simplified 2 model, including the effect of pressure change with time that Simplified 1 model does not consider. In order to vindicate the Rigorous model, two limiting cases without adsorption were proposed since the differences among different sets of the momentum and energy balance equations are in fact not relating to adsorption reaction. The mathematical models were solved using gPROMS software (Process System Enterprise Ltd 2012) coupling the mass, momentum and energy balance equations. The discretization method for the spatial domain in the column was orthogonal collocation on finite element method (OCFEM) with 100 intervals along the column.

\subsection{Very high pressure drop along the adiabatic column with no adsorption at steady-state}

This limiting case tackles fluid dynamics of a gas stream passing through an adiabatic, inert packed column with a very high pressure drop. It is well known that a throttling process where a fluid flows through a restriction, such as an orifice, a partly closed valve, or a porous plug, without any appreciable change in kinetic or potential energy, is isenthalpic. In case of ideal gas, the fluid temperature does not change during the throttling process (Smith et al. 2005). However, the gas temperature would change between the inlet and outlet of a throttling process if the gas flow through the throttling process involves a change of gas velocity.

The general energy balance for open system at steady state is

$\Delta\left[\left(H+\frac{1}{2} u^{2}+z g\right) \dot{m}\right]=\dot{Q}+\dot{W}_{S}$

Assuming negligible potential energy change, adiabatic condition, and no shaft work, Eq. (38) is simplified to

$\Delta\left(H+\frac{1}{2} u^{2}\right)=0$

Assuming ideal gas, the temperature change can be estimated by

$\Delta T=\frac{\left(\frac{1}{2} u_{\text {in }}^{2}-\frac{1}{2} u_{\text {out }}^{2}\right)}{\hat{C}_{p}}$

In order to look into the effect of the kinetic energy change incurred by pressure drop on the energy balance closely, we came up with a hypothetical case of inert feed stream of pure nitrogen at $400 \mathrm{~K}$ and 2 bar flowing at $1 \mathrm{~m} /$ $\mathrm{s}$ interstitial velocity at the inlet experiencing a very high pressure drop of 1 bar along the column in total. The column length is $0.5 \mathrm{~m}$. For simplicity, it was assumed that the column is adiabatic and the thermal axial dispersive flux is neglected. As it is well known that this process must be almost isenthalpic, the changes of temperature along the column as well as heat capacity are minimal. Assuming in this case the change of the gas velocity depends more on the pressure than the temperature, the outlet velocity was set to $2 \mathrm{~m} / \mathrm{s}$. Given the velocity change, the temperature change was estimated as $-1.426 \times 10^{-3} \mathrm{~K}$ using Eq. (40).

In this limiting case, the Rigorous model, Eq. (34), can be simplified to

$\rho_{g} \hat{C}_{p, g} \frac{d T}{d z}=\frac{d P}{d z}-f_{P D}$

Note that, in both Simplified 1 and 2 models, the RHS of Eq. (41) cancels out since the kinetic energy effect was not considered in their energy balance equations [see Eq. (26)]. As a result, there is no temperature change at all.

As expected, the Simplified 1 and 2 models could not predict any temperature change since the kinetic energy effect was neglected in the process of their derivation. On the contrary, the Rigorous model can estimate the outlet temperature of $399.9986 \mathrm{~K}$ which is in good agreement with the value calculated using Eq. (41) (Fig. 2).

\subsection{Blowdown of an adiabatic empty column}

The second limiting case was devised to see the effect of the term, $\varepsilon \frac{\partial P}{\partial t}$, on the energy balance for an empty column, i.e. $\varepsilon=1$, resulting in effectively no pressure drop along the column. Therefore, this limiting case is not to show the effect of kinetic energy but to verify that the Simplified 1 model cannot be used in simulating pressure-varying steps, such as pressurisation and blowdown. In this limiting case, a column, initially pressurised by inert nitrogen at 20 bar and $298 \mathrm{~K}$, is adiabatically depressurised to 1 bar. Again, the thermal axial dispersive flux was neglected for simplicity.

In this limiting case, the energy balance for open system can be written as (Smith et al. 2005):

$\frac{\partial(\rho \hat{U})}{\partial t}+\frac{\partial(\rho u \hat{h})}{\partial z}=0$

$\rho \frac{\partial \hat{h}}{\partial t}-\frac{\rho R}{\bar{M}} \frac{\partial T}{\partial t}+\hat{U} \frac{\partial \rho}{\partial t}+\frac{\partial(\rho u \hat{h})}{\partial z}=0 \quad$ (Ideal gas)

$\rho \frac{\partial \hat{h}}{\partial t}-\frac{\partial P}{\partial t}+\left(\hat{U}+\frac{R T}{\bar{M}}\right) \frac{\partial \rho}{\partial t}+\frac{\partial(\rho u \hat{h})}{\partial z}=0$

with $\hat{h}=\hat{U}+\frac{R T}{M}$ 

temperature profiles in the limiting case of a gas flow having very high pressure drop along the column among the three energy balances
Fig. 2 Comparison of the

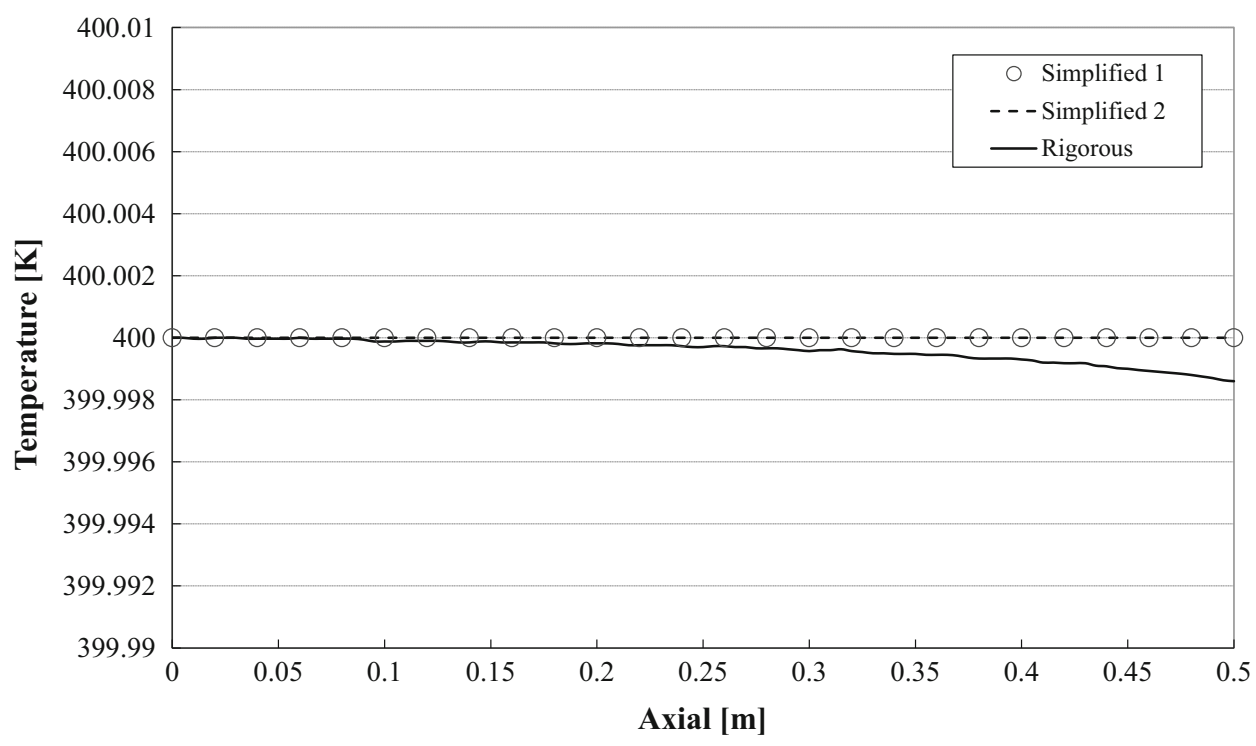

Fig. 3 Comparison of the temperature profiles at the outlet in the limiting case of the blowdown of an adiabatic empty column among the three energy balances

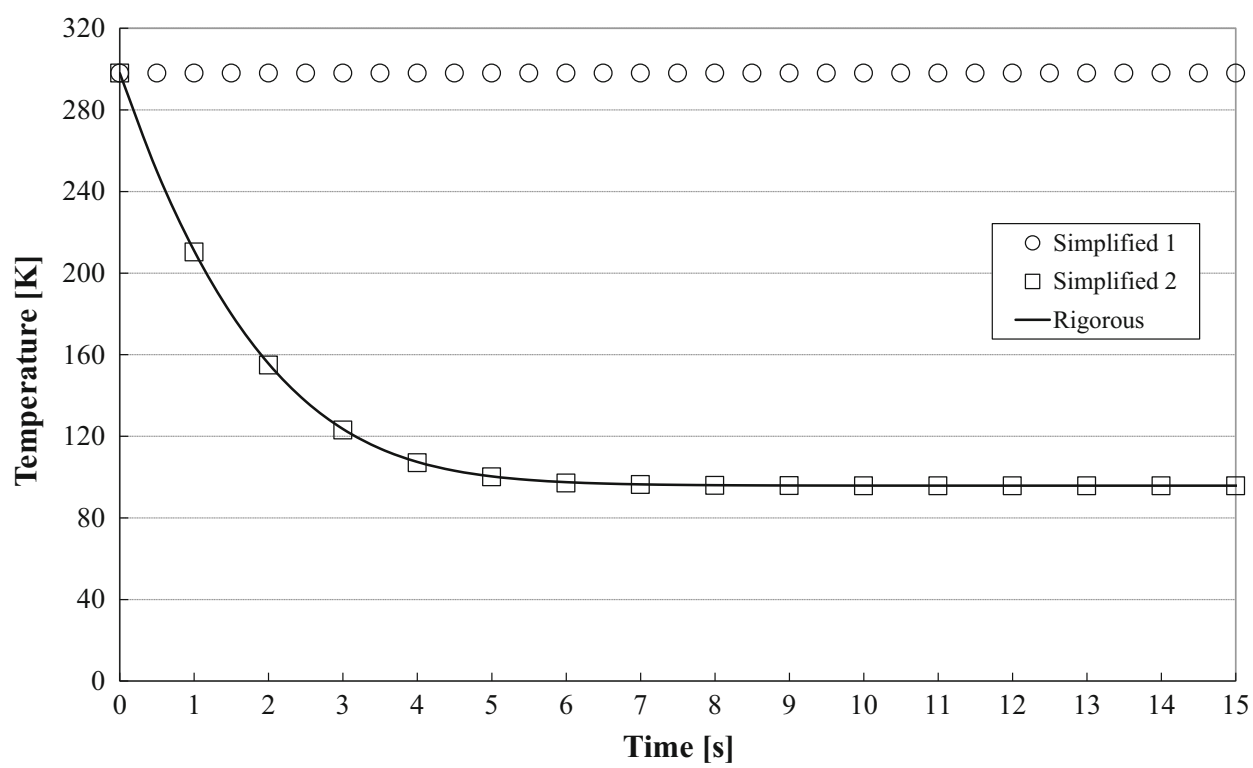

$\frac{\partial(\rho \hat{h})}{\partial t}-\frac{\partial P}{\partial t}+\frac{\partial(\rho u \hat{h})}{\partial z}=0$

Eq. (45) can also be obtained from the enthalpy balance in the gas phase by applying the simplifications of this limiting case to the Eq. (28). Finally the Eq. (45) can be converted to its equivalent energy balance equation in terms of temperature, Eq. (46), which can also be obtained from Eq. (31) by removing terms that are not necessary in this limiting case.

$\rho_{g} \hat{C}_{p, g, a v e}\left(\frac{\partial T_{g}}{\partial t}+u \frac{\partial T_{g}}{\partial z}\right)=\frac{\partial P}{\partial t}$
As shown in Fig. 3, the Simplified 1 model cannot predict the temperature change caused by the pressure decrease with time since it does not have the term on the RHS of Eq. (46). However, the Simplified 2 and Rigorous models can predict the identical final temperature of $95.6 \mathrm{~K}$ at the end of the blowdown operation.

\section{Conclusions}

A rigorous momentum and energy balance equations for adsorption process simulation were derived taking into account the kinetic energy change in temporal and spatial 
domains. Additional terms relating to the kinetic energy change were identified in comparison with commonly used momentum and energy balance equations that excluded the kinetic energy effects. Our rigorous energy balance was vindicated by the two limiting cases.

The effect of kinetic energy change on gas temperature may be negligible even for a gas flow system having a very high pressure drop. However, it is likely that the kinetic energy effect is amplified over the cycles of a Rapid Pressure Swing Adsorption (RPSA) process involving significant velocity change inside the adsorption column during its pressure-varying step, such as pressurisation and blowdown. It should be noted that as high pressure drop along the column has detrimental effects on adsorption process performance, use of structured adsorbents, e.g. monoliths, instead of pelletized adsorbents, has been considered to circumvent the pressure drop issue in RPSAs (Ahn and Brandani 2005a, b; Ritter 2014). However, pelletized adsorbents are still being used widely even for RPSAs since structured adsorbents are not always available for a variety of adsorption processes for gas separation. Therefore, the rigorous momentum and energy balances including kinetic energy effects will be useful in simulating a RPSA using pelletized adsorbents to evaluate the performance at its cyclic steady state more accurately.

Acknowledgments We would like to thank our colleagues at carbon capture group in the University of Edinburgh and Fei Chen (Air Products) for providing us with useful and insightful comments. Financial supports from KETEP (Grant No.: 2011-8510020030) and EPSRC (Grants No.: EP/F034520/1, EP/J018198/1, EP/J02077X/1 and EP/J020788/1) are greatly acknowledged.

Open Access This article is distributed under the terms of the Creative Commons Attribution 4.0 International License (http://creativecommons.org/licenses/by/4.0/), which permits unrestricted use, distribution, and reproduction in any medium, provided you give appropriate credit to the original author(s) and the source, provide a link to the Creative Commons license, and indicate if changes were made.

\section{Appendix}

According to the Aspen Adsim reference guide (2004), we can read the energy balance equations as follows taking into account only one dimensional case and neglecting the reaction terms.

For gas phase energy balance,

$$
\begin{gathered}
-\varepsilon k_{z, g} \frac{\partial^{2} T_{g}}{\partial z^{2}}+\varepsilon \rho_{g} u \hat{C}_{v g} \frac{\partial T_{g}}{\partial z}+\varepsilon_{B} \hat{C}_{v g} \rho_{g} \frac{\partial T_{g}}{\partial t}+P \frac{\partial v_{g}}{\partial z} \\
+h a_{p}\left(T_{g}-T_{s t}\right)+\frac{4 h_{w}}{D_{B}}\left(T_{g}-T_{w}\right)=0
\end{gathered}
$$

Note that by using $\varepsilon_{\mathrm{B}}$ in the third term of this equation, there is an implicit assumption that the gases in the macropore and the bulk fluid are at their thermal equilibrium.

For stationary phase energy balance,

$$
\begin{gathered}
-k_{z, s} \frac{\partial^{2} T_{s t}}{\partial z^{2}}+\hat{C}_{p s} \rho_{s} \frac{\partial T_{s t}}{\partial t}+\rho_{s} \sum_{i}\left(\hat{C}_{p a, i} w_{i}\right) \frac{\partial T_{s t}}{\partial t} \\
+\rho_{s} \sum_{i}\left(\Delta H_{i} \frac{\partial w_{i}}{\partial t}\right)-h a_{p}\left(T_{g}-T_{s t}\right)=0
\end{gathered}
$$

Assuming instantaneous thermal equilibrium between the gas and solid phases, the overall energy balance of Adsim reference guide becomes:

$$
\begin{gathered}
-k_{z, g} \varepsilon \frac{\partial^{2} T}{\partial z^{2}}-k_{z, s} \frac{\partial^{2} T}{\partial z^{2}}+\hat{C}_{p s} \rho_{s} \frac{\partial T}{\partial t}+\rho_{s} \sum_{i}\left(\hat{C}_{p a, i} w_{i}\right) \frac{\partial T}{\partial t} \\
+\rho_{s} \sum_{i}\left(\Delta H_{i} \frac{\partial w_{i}}{\partial t}\right)+C_{v g} v_{g} \rho_{g} \frac{\partial T}{\partial z}+\varepsilon_{B} \hat{C}_{v g} \rho_{g} \frac{\partial T}{\partial t} \\
+P \frac{\partial v_{g}}{\partial z}+\frac{4 h_{w}}{D_{B}}\left(T-T_{w}\right)=0
\end{gathered}
$$

Finally, the Rigorous model, Eq. (34), can be converted into a formula similar to the Adsim equation, Eq. (49).

$$
\begin{aligned}
\varepsilon \rho_{g} \hat{C}_{v g, a v e}\left(\frac{\partial T}{\partial t}+u \frac{\partial T}{\partial z}\right) \\
\quad+(1-\varepsilon)\left[\rho_{s} \hat{C}_{p s} \frac{\partial T}{\partial t}+\rho_{a} \hat{C}_{p g, a v e} \frac{\partial T}{\partial t}-\sum_{i}^{N_{c}}\left(-\Delta H_{i}\right) \frac{\partial q_{i}}{\partial t}\right] \\
\quad=\varepsilon k_{z, g} \frac{\partial^{2} T}{\partial z^{2}}-\varepsilon P \frac{\partial u}{\partial z}+\frac{R T}{\bar{M}} \Gamma+\varepsilon u f_{P D}-\varepsilon \frac{4 h_{w}}{D_{B}}\left(T-T_{w}\right)
\end{aligned}
$$

Comparing the alternative form of Rigorous model, Eq. (50), with the Adsim overall energy balance, Eq. (49), the missing terms in the Adsim energy balances are $\frac{R T}{M} \Gamma$ and $\varepsilon u f_{P D}$.

Applying this model just as it is presented in the reference guide to the limiting case of "very high pressure drop along the adiabatic column with no adsorption at steadystate', strange behaviour can be observed. In this limiting case, the Adsim energy balance, Eq. (49), is simplified into:

$$
\begin{aligned}
C_{v g} u \rho_{g} \frac{d T}{d z}+P \frac{d u}{d z} & =0 \quad \text { or } \\
C_{p g} \rho_{g} \frac{d T}{d z}-\frac{d P}{d z} & =0(\text { at steady }- \text { state, no adsorption })
\end{aligned}
$$

Therefore, it is very likely that the Adsim equation simply neglected energy dissipation term relating to pressure drop even though the term of pressure (or velocity) change along the column incurred by the kinetic energy change was included. This explanation seems farfetched but Eq. (51) cannot be explained in other ways. It should be noted that an abnormal temperature drop of 
Fig. 4 Comparison of temperature profiles in the limiting case of a gas flow having a very high pressure drop along the column between actual Adsim run and gPROMS simulation using the energy balance formula in Adsim reference guide

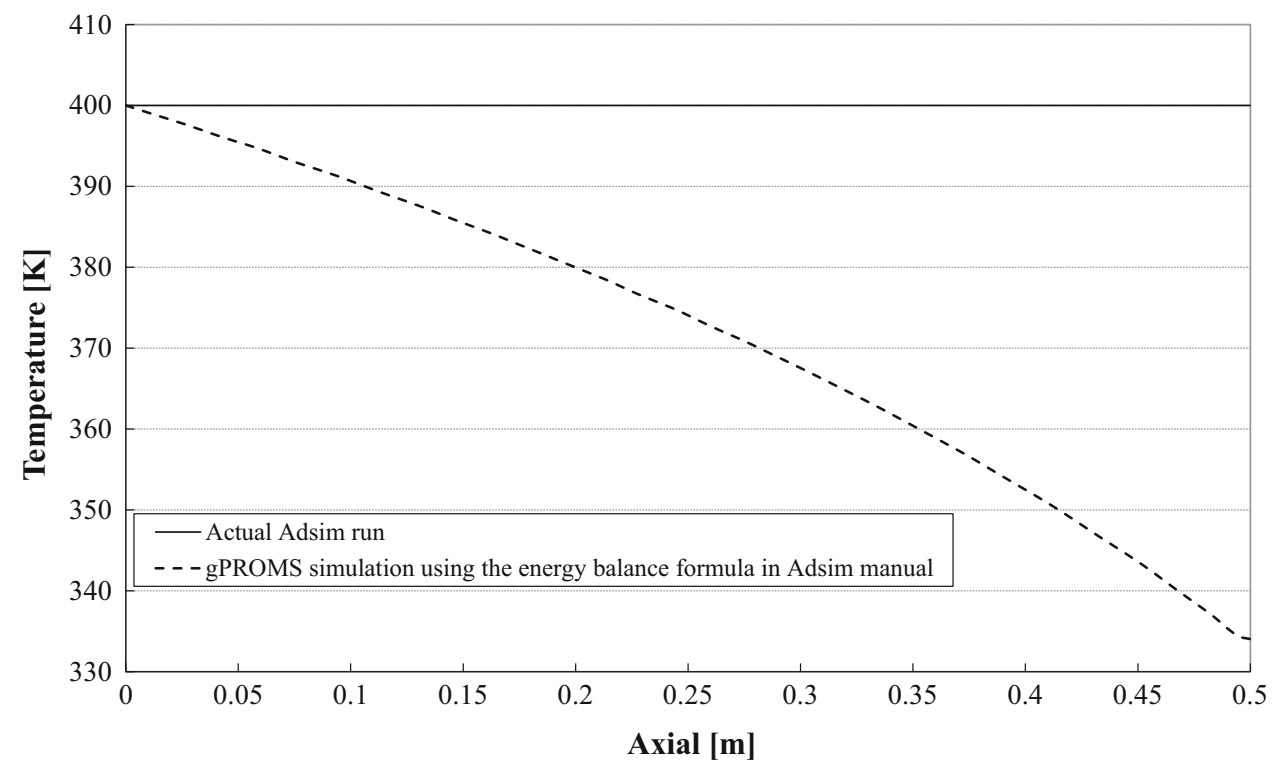

almost $70 \mathrm{~K}$ was obtained with the Adsim equation (Fig. 4). Nevertheless, by simulating the same limiting case with the actual Adsim software no temperature change was observed as shown in Fig. 4.

In the other limiting case of the 'blowdown of the adiabatic column with no pressure drop and no adsorption', the actual Adsim software generated the same result as those by the Simplified 2 and Rigorous models [Eq. (46)]. It can be concluded that the energy balance equations written in the Adsim reference guide should be corrected as the energy balance equation actually being used in the software must be the same as the Simplified 2 model.

\section{References}

Adsim, A.: Adsorption Reference Guide. Aspen Technology Inc., Cambridge (2004)

Ahn, H., Lee, C.-H., Seo, B., Yang, J., Baek, K.: Backfill cycle of a layered bed $\mathrm{H}_{2}$ PSA process. Adsorption 5, 419-433 (1999)

Ahn, H., Brandani, S.: Analysis of breakthrough dynamics in rectangular channels of arbitrary aspect ratio. AIChE J. 51, 1980-1990 (2005a)

Ahn, H., Brandani, S.: Dynamics of carbon dioxide breakthrough in a carbon monolith over a wide concentration range. Adsorption 11, 473-477 (2005b)

Da Silva, F.A., Silva, J.A., Rodrigues, A.E.: A general package for the simulation of cyclic adsorption processes. Adsorption 5, 229-244 (1999)

Da Silva, F.A., Rodrigues, A.E.: Propylene/propane separation by vacuum swing adsorption using $13 \mathrm{X}$ zeolite. AIChE J. 47, 341-357 (2001)

Farzaneh, H., Ghalee, I., Dashti, M.: Simulation of a multifunctional energy system for cogeneration of steam, power and hydrogen in a coke making plant. Procedia Environ. Sci. 17, 711-718 (2013)
Huang, Q., Malekian, A., Eic, M.: Optimization of PSA process for producing enriched hydrogen from plasma reactor gas. Gas Sep Purif. 62, 22-31 (2008)

Ishii, M., Hibiki, T.: Thermo-Fluid Dynamics of Two-Phase Flow, 2nd edn, pp. 437-448. Springer, New York (2010)

Kikkinides, E.S., Yang, R.T.: Concentration and recovery of $\mathrm{CO}_{2}$ from flue gas by pressure swing adsorption. Ind. Chem. Eng. Res. 32, 2714-2720 (1993)

Kim, M.-B., Bae, Y.-S., Choi, D.-K., Lee, C.-H.: Kinetic separation of landfill gas by a two-bed pressure swing adsorption process packed with carbon molecular sieve: non-isothermal operation. Ind. Chem. Eng. Res. 45, 5050-5058 (2006)

Kostroski, K.P., Wankat, P.C.: High recovery cycles for gas separations by pressure-swing adsorption. Ind. Eng. Chem. Res. 45, 8117-8133 (2006)

Lu, Z.P., Loureiro, J.M., LeVan, M.D., Rodrigues, A.E.: Dynamics of pressurization and blowdown of an adiabatic adsorption bed: III Gas Sep Purif. 6, 15-23 (1992a)

Lu, Z.P., Loureiro, J.M., LeVan, M.D., Rodrigues, A.E.: Dynamics of pressurization and blowdown of an adiabatic adsorption bed: IV. Intraparticle diffusion/convection models. Gas Sep Purif. 6, 89-100 (1992b)

Mahle, J.J., Friday, D.K., LeVan, M.D.: Pressure swing adsorption for air purification. 1. Temperature cycling and role of weakly adsorbed carrier gas. Ind. Eng. Chem. Res. 35, 2342-2354 (1996)

Process System Enterprise Ltd.: (2012) www.psenterprise.com

Reynolds, S.P., Ebner, A.D., Ritter, J.A.: Stripping PSA cycles for $\mathrm{CO}_{2}$ recovery from flue gas at high temperature using a hydrotalcite-like adsorbent. Ind. Chem. Eng. Res. 45, 4278-4294 (2006)

Ribeiro, A.M., Grande, C.A., Lopes, F.V.S., Loureiro, J.M., Rodrigues, A.E.: A parametric study of layered bed PSA for hydrogen purification. Chem. Eng. Sci. 63, 5258-5273 (2008)

Richard, M.A., Benard, P., Chahine, R.: Gas adsorption process in activated carbon over a wide temperature range above the critical point. Part 2: conservation of mass and energy. Adsorption 15, 53-63 (2009)

Ritter, J.A.: Bench-scale development and testing of rapid PSA for $\mathrm{CO}_{2}$ capture. In: 2014 NETL $\mathrm{CO}_{2}$ Capture Technology Meeting, Pittsburgh (2014) 
Rodrigues, A.E., Loureiro, J.M., LeVan, M.D.: Simulated pressurization of adsorption beds Gas Sep Purif. 5, 115-124 (1991)

Ruthven, D.M.: Principles of Adsorption and Adsorption Processes. Wiley, New York (1984)

Ruthven, D.M., Farooq, S., Knaebel, K.S.: Pressure Swing Adsorption. VCH, New York (1994)

Sereno, C., Rodrigues, A.E.: Can steady-state momentum equations be used in modelling pressurization of adsorption beds? Gas Sep. Purif. 3, 167-174 (1993)

Sharma, P.K., Wankat, P.C.: Hybrid cycles to purify concentrated feeds containing a strongly adsorbed impurity with a nonlinear isotherm: the PSA-TSA super-cycle. Ind. Eng. Chem. Res. 48, 6405-6416 (2009)

Smith, J.M., Van Ness, H.C., Abbott, M.B.: Introduction to Chemical Engineering Thermodynamics. McGraw-Hill, New York (2005)

Suzuki, M.: Adsorption Engineering. Kodansha, Tokyo (1990)

Walton, K.S., LeVan, M.D.: Effect of energy balance approximations on simulation of fixed-bed adsorption. Ind. Eng. Chem. Res. 44, 7474-7480 (2005)

Yang, R.T.: Gas Separation by Adsorption Processes. Butterworth, Boston (1987) 